\title{
Protocolo para a distribuição de informações de canais em redes em malha sem fio
}

\author{
Roni Francis Shigueta ${ }^{1}$ \\ Hermes Nuss ${ }^{1}$ \\ Paulo Henrique Aguiar ${ }^{1}$ \\ Alexandre Ferreira dos Santos ${ }^{1}$
}

\begin{abstract}
Resumo: Este artigo apresenta um protocolo para a distribuição de informações de canais em uma rede em malha sem fio. $\mathrm{O}$ canal corresponde à faixa de frequência na qual dois dispositivos sem fio (nós da rede) podem se comunicar. O protocolo apresentado permite criar um repositório que contém os canais que estão disponíveis em um dispositivo para que se comunique com o seu nó vizinho. A partir das informações desse repositório, os dispositivos podem tomar decisões de alocação de canais, baseadas em algum critério, como por exemplo, a menor interferência gerada na rede. Dependendo da quantidade de canais disponíveis, é possível atribuir canais diferentes aos enlaces da rede (par de nós), permitindo transmissões simultâneas. A simulação do protocolo é realizada por meio de canais de frequência do padrão IEEE 802.11b. Apesar das simulações serem realizadas nesse padrão, o protocolo pode ser adaptado para operar em outras faixas de frequência. Os resultados demonstram que a partir dos valores dos canais transmitidos através de mensagens de hello, é possível encontrar os canais comuns entre dois nós, realizando a intersecção entre a lista de canais do nó vizinho com a lista do nó local.
\end{abstract}

Palavras-chave: Canais de frequência. Padrão IEEE 802.11. Rede sem fio.

\begin{abstract}
This article presents a protocol for the distribution of channel information in a wireless mesh network. The channel corresponds to the frequency range in which two wireless devices (network nodes) can communicate. The proposed protocol allows create a repository that contains the channels that are available in a device so that it can communicate with its neighbor. From this information repository, the devices can take channel allocation decisions based on some criteria, for example, less interference generated in the network. Depending on the number of available channels, is a possible assign different channel to network links (two nodes) allowing simultaneous transmissions. The simulation of the protocol is performed using frequency channels of IEEE $802.11 \mathrm{~b}$ standard. Although simulations are performed in this pattern, the protocol can be adapted to operate in other frequency bands. The results show that from the values of channels transmitted through hello messages, it is possible to find common channels between two nodes performing the intersection of the neighbor channel list with the local node list.
\end{abstract}

Keywords: Frequency channels. IEEE 802.11 standard. Wireless network.

\section{Introdução}

Este artigo apresenta um protocolo para a distribuição de informações de canais em uma rede em malha sem fio. Tal tipo de rede corresponde a uma rede em que os roteadores são estáticos ou possuem pouca mobilidade [1]. Uma das funções do roteador é encaminhar os pacotes até que cheguem ao seu destino. A decisão de qual roteador é utilizado para encaminhar o pacote é tomada com base em rotas que são obtidas de

\footnotetext{
${ }^{1}$ Curso de Tecnologia em Análise e Desenvolvimento de Sistemas. UNIPAR, Avenida Brasil 1123 - Cianorte (PR) - Brasil \{roni@unipar.br, hermesnuss@hotmail.com,p.aguiar@live.com, xandykalo@hotmail.com\}
}

http://dx.doi.org/10.5335/rbca.2014.3339 
uma tabela de roteamento. As rotas são inseridas na tabela, utilizando roteamento estático (manual) ou utilizando um protocolo de roteamento que calcula as rotas dinamicamente.

A Figura 1 ilustra a representação de uma rede em malha. A rede é constituída de sete roteadores, que são representados pelas antenas localizadas no ponto mais alto das edificações. A comunicação entre dois roteadores é realizada pelo enlace de comunicação. Para que o enlace esteja ativo, é necessário que os dois roteadores estejam sintonizados no mesmo canal de frequência. Na Figura1, os roteadores estão localizados em posições estáticas, caracterizando a natureza da rede em malha.

Figura 1: Rede em malha

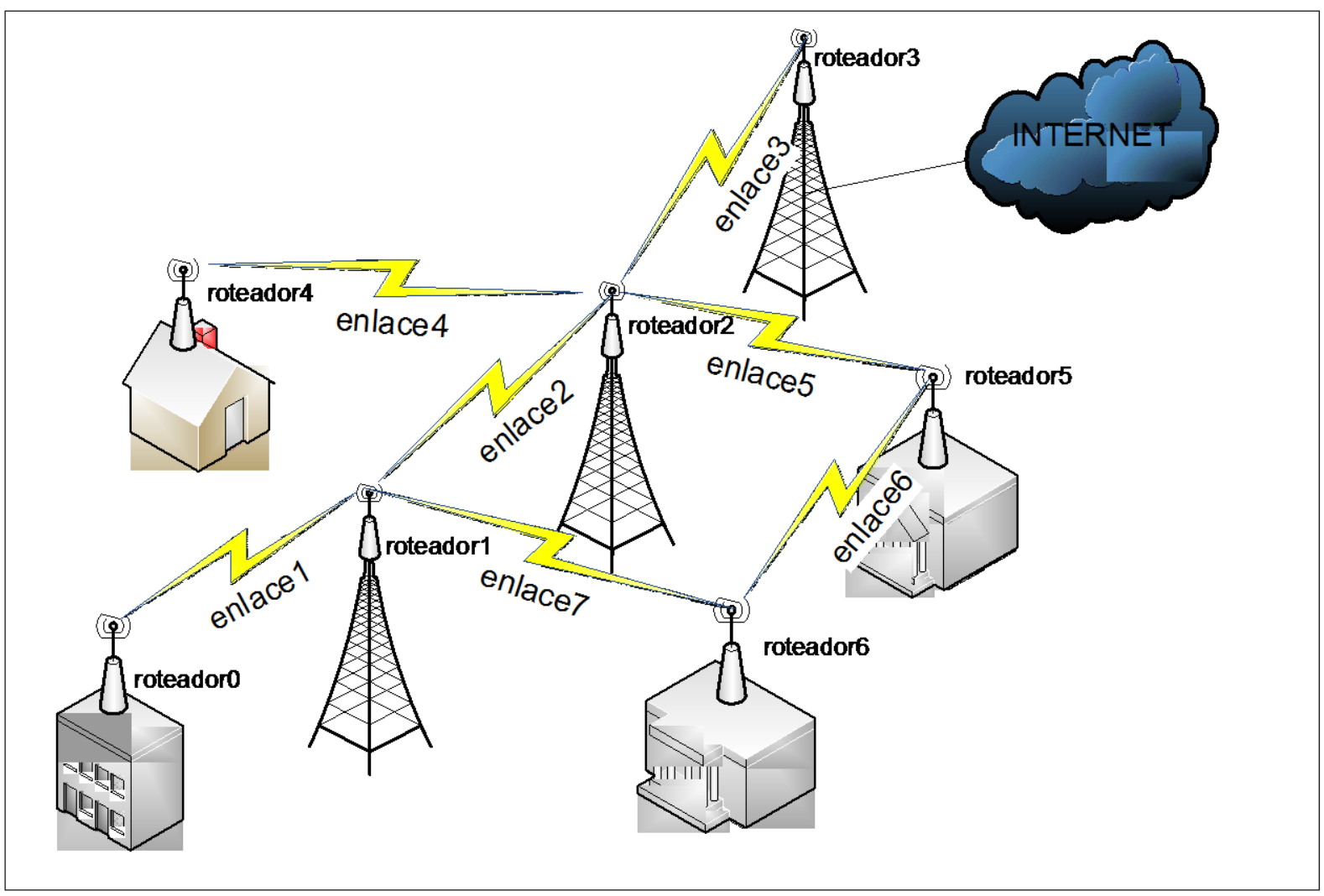

Para que seja possível a comunicação do roteador0 com a internet, é necessário que o tráfego passe por roteadores intermediários. A quantidade de roteadores que um pacote percorre define a quantidade de saltos (hops) entre um roteador de origem e destino. A quantidade de saltos permite definir a distância entre o roteador de origem e de destino. Na Figura 1, a distância entre o roteador0 e o roteador3 depende do caminho (rota) que o pacote percorrerá. No caso, há dois caminhos possíveis, ou utilizando o roteador2 ou o roteador6. Caso o pacote utilize a rota pelo roteador2, haverá uma distância de três saltos. Caso utilize a rota pelo roteador6, a distância do caminho aumenta para cinco saltos.

Para se representar uma rede como a da Figura 1, é utilizada a notação da teoria dos grafos. Utilizando essa notação, a rede é representada por um grafo no qual os roteadores correspondem aos nós e os enlaces de comunicação correspondem às arestas do grafo [2]. Dois nós são interligados por meio de uma aresta no grafo. A aresta utiliza um canal comum aos dois nós.

O canal corresponde à faixa de frequência de rádio que um nó utiliza para se comunicar com o seu nó vizinho. Para que haja a comunicação entre dois nós, é necessário que ambos utilizem o mesmo canal de frequência. Porém, se houver um único canal compartilhado em toda a rede, no momento em que um determinado canal está sendo utilizado, os demais nós na vizinhança devem aguardar a liberação desse canal. 
Caso um nó transmita pacotes em um canal que já está sendo utilizado, ocorrerão colisões, sendo necessária a retransmissão dos pacotes, diminuindo a vazão da rede. As tecnologias de acesso das redes sem fio baseadas no padrão IEEE 802.11b utilizam um único canal compartilhado na rede. Dessa forma, quando um nó IEEE $802.11 \mathrm{~b}$ deseja transmitir, deve aguardar a liberação do canal.

O protocolo apresentado procura extender o protocolo $802.11 \mathrm{~b}$ através da distribuição das informações dos canais que estão disponíveis nos nós da rede.

Com essas informações, cada nó passa a ter um repositório contendo os canais que estão disponíveis para se comunicar com o seu nó vizinho, possibilitando a comunicação simultânea de pares de nós. Na seção 2 , é apresentado os canais do padrão IEEE $802.11 \mathrm{~b} / \mathrm{g}$. Na 3, encontram-se alguns trabalhos relacionados. Na seção seguinte, é exposto o modelo do protocolo. A seção 5 apresenta o cenário de simulação. Na 6 , demonstra-se os resultados e, por fim, na seção 7, é apresentada a conclusão.

\section{Canais do padrão IEEE 802.11b/g}

De acordo com Gast [3], existem três faixas de canais de frequências que podem ser utilizadas livremente sem a necessidade de obtenção de uma licença exclusiva junto à operadora de telecomunicações. Essas faixas de frequência são chamadas de faixa ISM (Industrial, Scientific and Medical) e correspondem às bandas de 902-928 $\mathrm{MHz}, 2.4-2.5 \mathrm{GHz}$ e $5.725-5.875 \mathrm{GHz}$.

O padrão IEEE 802.11a utiliza a faixa de frequência de 5.725-5.875 GHz, possibilitando uma taxa de transferência de dados de até $54 \mathrm{Mbps}$. Os padrões IEEE 802.11b e IEEE 802.11g utilizam a faixa de frequência de 2.4-2.5 GHz com taxas de transferências de até $11 \mathrm{Mbps}$ e $54 \mathrm{Mbps}$ respectivamente. Neste trabalho, é utilizado o padrão IEEE $802.11 \mathrm{~b} / \mathrm{g}$, pois é o padrão utilizado no Brasil. A Fig. 2 ilustra a distribuição dos canais de frequências para o padrão IEEE $802.11 \mathrm{~b} / \mathrm{g}$ [4].

Figura 2: Canais de frequência do padrão IEEE $802.11 \mathrm{~b} / \mathrm{g}$

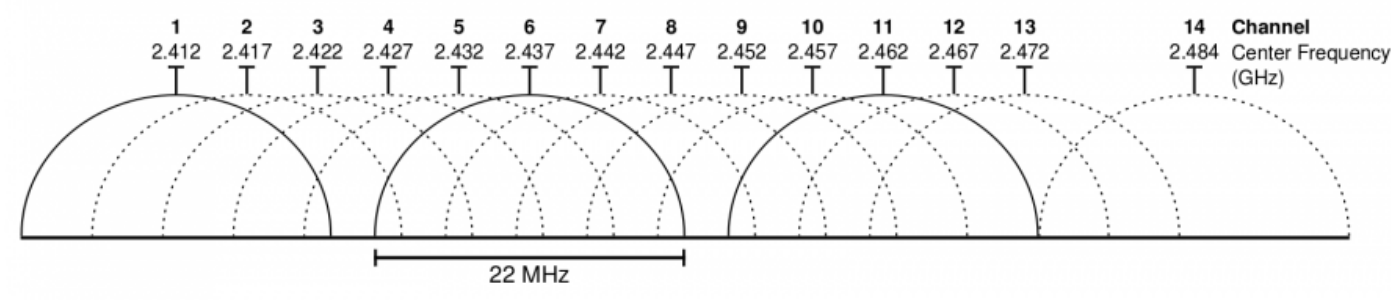

Como se pode observar na Figura 2, o padrão IEEE $802.11 \mathrm{~b}$ possui 14 canais. Cada canal possui um número que representa a frequência central do canal e uma largura de banda de $22 \mathrm{MHz}$. A distância entre as frequências centrais é de $5 \mathrm{MHz}$, ocorrendo a sobreposição de canais adjacentes o que causa a interferência entre canais.

Os canais 2, 3, 4 e 5 são interferentes com o canal 1. Pode-se observar na figura que as extremidades das bandas dos canais 1 e 5 se sobrepõem, causando interferência nesses pontos. Para que isso não ocorra entre os canais vizinhos (interferência de canal adjacente), é necessário que haja uma distância de no mínimo $25 \mathrm{MHz}$ entre as frequências centrais dos canais. Dessa forma, os canais são distribuídos obedecendo a essa distância mínima, permitindo que, por exemplo, os canais 1,6 e 11 sejam utilizados simultaneamente sem que ocorra interferência entre eles.

Além da interferência de canal adjacente, existe a interferência causada pela atribuição do mesmo canal a enlaces vizinhos (interferência de co-canal). A Figura 3 ilustra uma rede com quatro nós, dois fluxos simultâneos (fluxo de dados 1 e fluxo de dados 2) e um único canal disponível na rede (canal 6). 
Figura 3: Rede com dois fluxos simultâneos utilizando um único canal

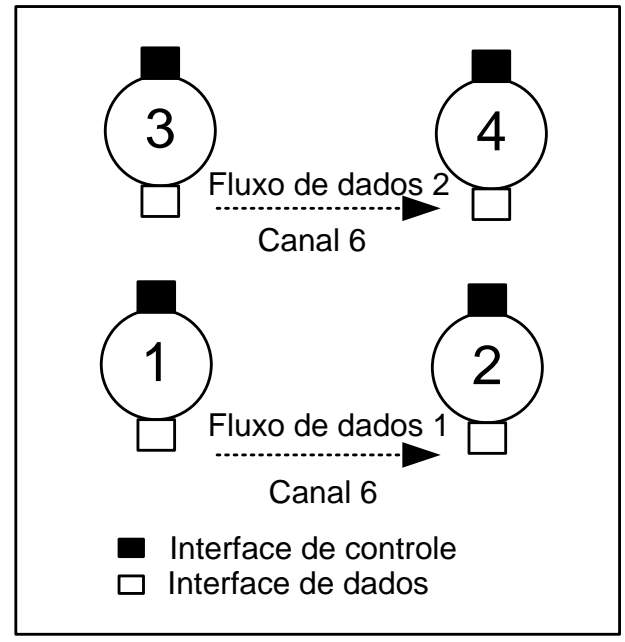

Pode-se observar que o nó 1 envia um fluxo de dados (fluxo de dados 1) para o nó 2, por intermédio do canal 6. O nó 3 envia um fluxo de dados para o nó 4 (fluxo de dados 2) utilizando, também, o canal 6. Assim, ao fazer uso de um mesmo canal ao mesmo tempo, ocorrerá a interferência de um fluxo no outro. Nesse caso, ocorrerão colisões, perda de pacotes e, consequentemente, será necessário retransmiti-los, fazendo a vazão da rede diminuir.

A criação de um protocolo que disponibilize um repositório de canais comuns permitirá que cada nó tenha mais de uma opção de canal para se comunicar com o seu vizinho. A Figura 4 ilustra um exemplo de utilização de um repositório de canais, o qual consiste em uma lista de canais aos quais um nó pode recorrer para se comunicar com seu vizinho e é representado na figura pelos números dos canais entre chaves.

Figura 4: Rede com dois fluxos simultâneos utilizando dois canais diferentes

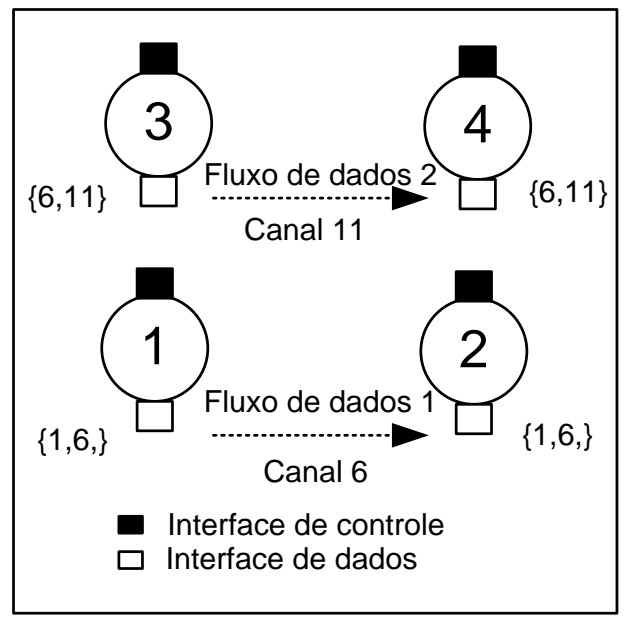

A Figura 4 ilustra que o nó 1 tem duas opções de canais (canal 1 ou 6 ) para se comunicar com o nó 2 , decidindo utilizar o canal 6 . O nó 3 , também, possui duas opções para se comunicar com o nó 4 (canal 6 ou 11) optando pelo canal 11. Como os dois fluxos utilizam canais diferentes que são ortogonais entre si (sem interferência dos canais vizinhos), um fluxo não interferirá no outro. Além disso, ao utilizarem canais diferentes, os dois fluxos podem transmitir simultaneamente, aumentando a vazão da rede. 


\section{Trabalhos relacionados}

$\mathrm{Na}$ literatura, vários autores tratam dos problemas de canais em redes sem fio, principalmente, com relação à interferência entre canais.

Em Hossain e Niyato [5], é abordada a questão de alocação dos canais em função da temperatura de interferência que corresponde ao monitoramento de um canal de frequência para verificar se esse possui sinais, interferentes ou não. Em função da qualidade do canal avaliado, toma-se a decisão de se transmitir ou não nesse canal.

Em Khalife e Ahuja [6], é tratada a seleção de canais, baseada na distribuição de interferência em cada canal, atribuindo-lhe um peso de acordo com a probabilidade da capacidade do enlace do canal ser maior do que a demanda de envio de dados. O algoritmo utiliza múltiplas frequências entre nós de origem e destino para satisfazer uma determinada demanda de capacidade de tráfego. Além disso, ocorre a agregação de canais de frequências diferentes e a transmissão simultânea de dados a fim de aumentar a largura de banda da rede. Nesse caso, são utilizadas várias interfaces de rádio, cada uma representando um canal de frequência diferente do que foi utilizado na agregação.

Em Subramanian e Gupta [2], é abordado o modelo de interferência baseado em grafo de conflitos. Em seu trabalho, os autores modelam a rede como um grafo de comunicação, transformando-o em um grafo de conflito. O primeiro representa os nós das redes interligados aos seus vizinhos (vizinhos a 1 salto), já no segundo, cada um de seus nós corresponde a um enlace (par de nós) do grafo de comunicação. Dessa forma, este representa a interligação entre nós e um grafo de conflito representa a interligação de enlaces da rede. Uma vez determinado o grafo de conflito, o autor define o modelo de interferência no qual dois enlaces de um grafo de comunicação são considerados interferentes se dois nós vizinhos no grafo de conflito utilizar o mesmo canal ao mesmo tempo. Outra forma de se definir esse modelo de interferência é que ocorrerá interferência se os vizinhos a dois saltos de um nó em um grafo de comunicação utilizarem o mesmo canal ao mesmo tempo.

Em Hurley e Smith [7], são apresentadas várias técnicas para a atribuição de canais em redes sem fio. Os autores abordam técnicas meta-heurísticas como algoritmos genéticos, anelamento simulado e busca tabu. As técnicas meta-heurísticas trabalham com aproximações para solucionar problemas de otimização combinacional que são difíceis de resolver.

Em Shin, Seungjoon e Kim [8], é apresentado um modelo de atribuição de canais de forma distribuída em redes sem fio, utilizando múltiplas interfaces de rádio. Tal forma permite que cada nó da rede tome sua própria decisão sobre o canal a ser utilizado. Os autores valem-se de um modelo de rede para diminuir a interferência a dois saltos e sua métrica de avaliação corresponde à vazão e atraso obtidos por meio de um simulador de redes. Shin, Seungjoon e Kim, ainda, comparam uma atribuição aleatória de canais com uma atribuição supervisionada, na qual acrescenta a restrição de que o número de canais é menor ou igual a duas vezes o número de interfaces menos um. Essa restrição permite garantir que haja pelo menos um canal disponível para a transmissão/recepção de dados.

Em Zheng [9], é apresentada a atribuição de canais baseada em um modelo de fluxo máximo. No trabalho, o objetivo é diminuir o número de comutação (mudança) de canais no decorrer das rotas que constituem um fluxo, de modo a reduzir o tempo de comutação. Para tanto, são empregadas como métricas, a vazão da rede e o atraso fim a fim. Além disso, os autores comparam o modelo de fluxo com o baseado na atribuição de canais a cada enlace, demonstrando que o seu modelo resulta em uma vazão, aproximadamente, $10 \%$ maior e um atraso fim a fim 50\% menor. Como o modelo proposto por Zheng [9] prioriza a diminuição da comutação de canais, podem ocorrer interferências devido aos enlaces vizinhos, pertencentes ao mesmo fluxo, utilizarem o mesmo canal.

Em Walenga et al. [10], é apresentado um algoritmo para atribuir canais aos nós em uma rede sem fio e mitigar a interferência. Os autores apresentam uma rede em malha cognitiva na qual os nós possuem duas interfaces de redes. A primeira interface é utilizada como controle e a segunda como interface de dados. O modelo de interferência aplicado é o de grafo de conflito a dois saltos, utilizando a interferência reduzida como métrica. Essa métrica fornece uma relação entre a quantidade de pares de enlaces que interferem entre si e a interferência máxima na rede devido à utilização de um único canal. Os autores realizam uma comparação entre seu algoritmo com outros dois, um centralizado e outro aleatório. O centralizado representa a melhor situação de atribuição com menor interferência, porém com um overhead maior na rede. Nesse caso, o overhead 
corresponde à quantidade de mensagens de controle na rede que foram necessárias para o algoritmo convergir. $\mathrm{O}$ algoritmo aleatório representa a pior situação. Considerando a métrica da interferência reduzida, Walenga, et al. demonstram que o algoritmo proposto apresenta resultados bem próximos do algoritmo centralizado, porém com menor overhead.

\section{Modelo do protocolo}

Conforme ilustra a Figura 5, cada nó de uma rede em malha possui duas interfaces de rádio. A primeira é utilizada para as operações de controle da rede, sendo responsável pelo envio e recepção das mensagens de hello, as quais são implementadas na camada de aplicação, tornando-a adaptável aos protocolos de mais baixo nível existentes. A segunda interface é responsável pelo tráfego de dados. As interfaces de controle e de dados são configuradas com canais e endereços de rede diferentes. Como o canal da interface de controle é diferente dos canais da interface de dados, os pacotes gerados pela rede de controle não influenciam na rede de dados.

Figura 5: Rede em malha com duas interfaces de redes em cada nó

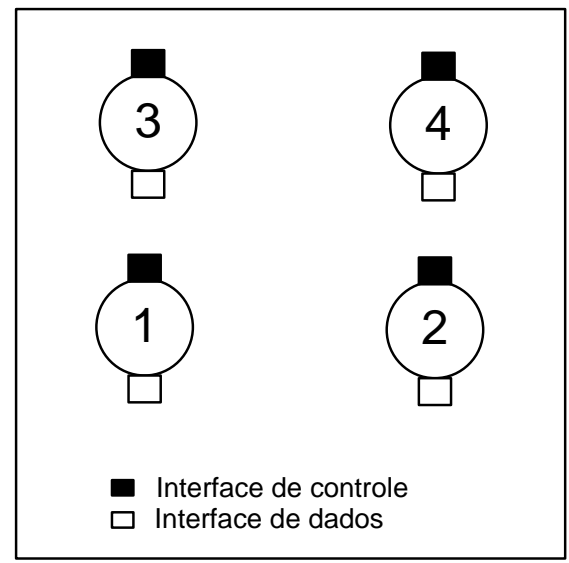

As mensagens de hello são responsáveis pela construção da topologia da rede e pela troca de informações sobre quais canais estão disponíveis em cada nó. O intervalo de troca das mensagens é de 2 segundos. A Figura 6 ilustra a estrutura da mensagem de hello. A mensagem possui um cabeçalho de pacote que contém o endereço IP da máquina que está enviando a mensagem. O campo número de canais locais contém a quantidade de canais de frequência disponíveis no nó de origem. O campo tamanho da mensagem define o tamanho em bytes da mensagem. O campo de canal local corresponde ao valor do canal disponível. Pode-se observar na Figura 6 que cada campo de canal local possui 8 bits, possibilitando números de identificação de canais de 0 a 255 . Esses números de canais podem ser facilmente mapeados para a faixa de frequência desejada, porém, neste trabalho, os números dos canais correspondem aos mesmos números de canais do padrão IEEE 802.11b.

Figura 6: Estrutura da mensagem de hello

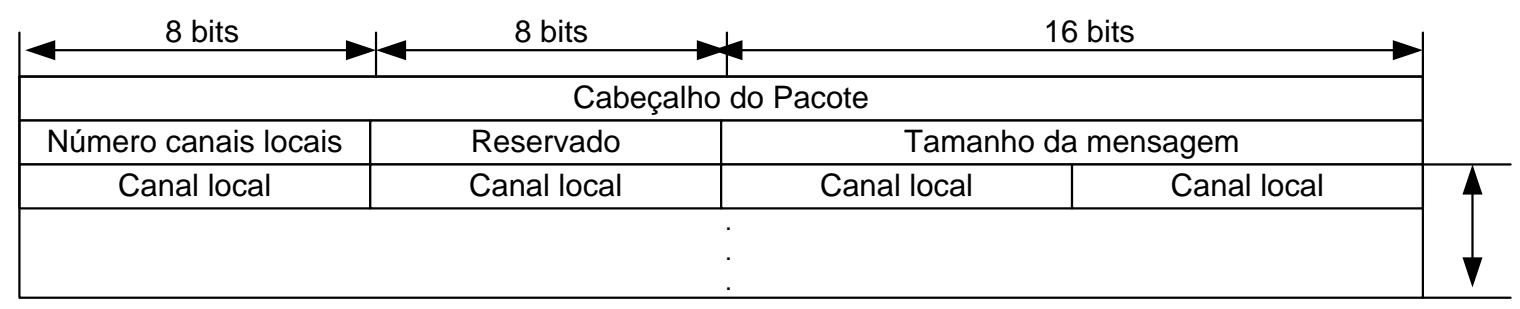

Ao receber a mensagem de hello, o nó local retira as informações dos canais disponíveis da mensagem e realiza uma intersecção com seus canais locais. Dessa intersecção, resulta a lista de canais disponíveis para a comunicação. 
O pseudocódigo a seguir representa os métodos que foram utilizados no protocolo, o qual é constituído de 3 métodos: EnviarHello(), ProcessarHello() e AtualizarRepositorio() .

O método EnviarHello( ) é responsável por adicionar o número de canais disponíveis e o valor de cada um desses canais no corpo da mensagem. Além disso, o método adiciona o endereço IP do nó no cabeçalho anexando-o ao corpo da mensagem. Ao término da montagem da mensagem de hello, essa é colocada no buffer para ser enviado na rede.

O método ProcessarHello( ) é responsável por ler cada mensagem de hello recebida. Ao receber a mensagem, é retirado o endereço IP do cabeçalho e colocado numa lista. O endereço IP da mensagem, juntamente, com o endereço IP do nó que recebeu a mensagem formam o enlace. Após essa formação, é retirado cada um dos canais da mensagem e colocado na lista de canais.

O método AtualizarRepositorio( ) é responsável por ler a lista de canais gerada pelo método ProcessarHello( ) e comparar cada um dos canais da lista com os canais do nó local. Se o canal da lista for igual ao canal do nó, significa que os dois nós possuem um canal em comum. Nesse caso, o canal é adicionado ao repositório.

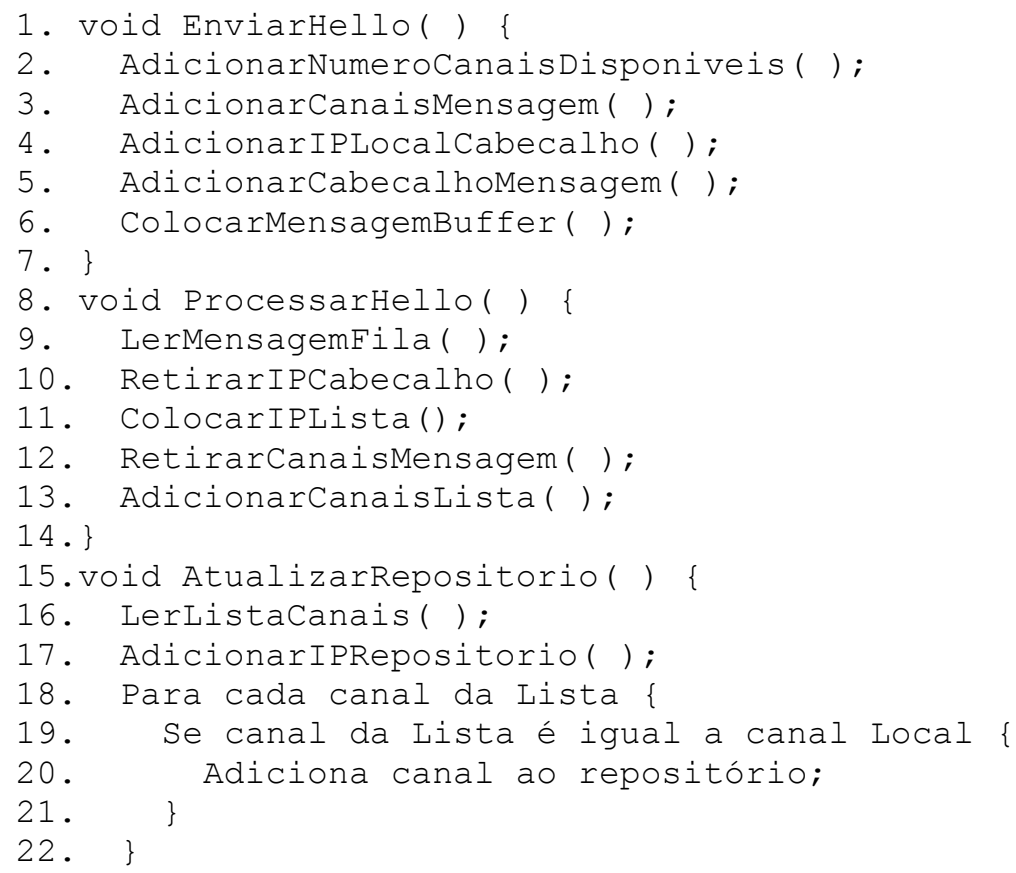

A Figura 7 ilustra a máquina de estados de cada nó em relação à sua interface de controle e ao protocolo. Há três estados possíveis em que o nó pode estar: enviando a mensagem, processando, ou atualizando o repositório de canais. Para mudar de estado, é necessário que o protocolo execute um dos seus métodos. 
Figura 7: Máquina de estados da interface de controle

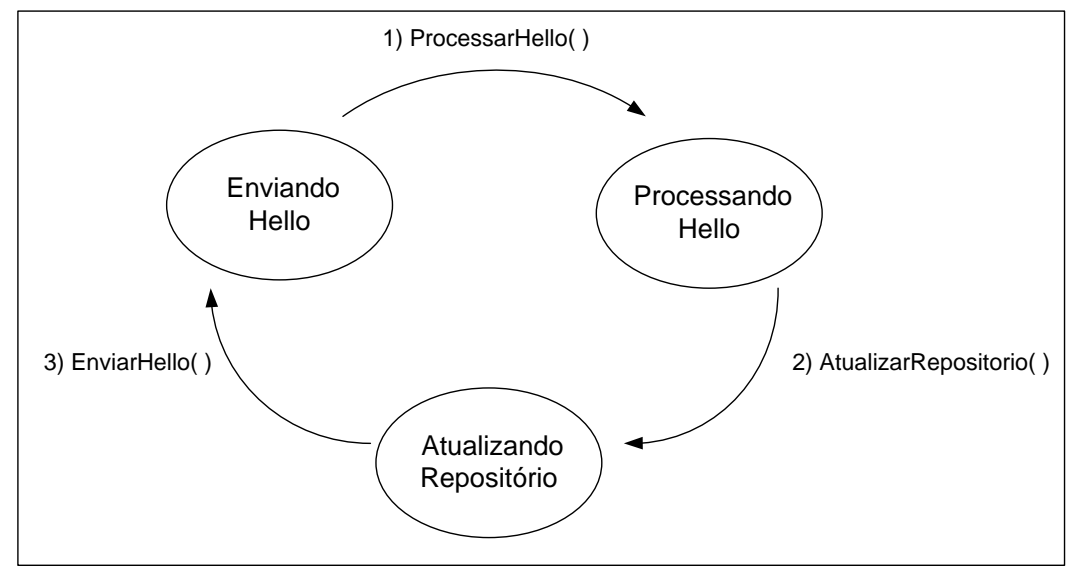

\section{Cenário de simulação}

Para a simulação, foi utilizado o simulador de redes NS-3, utilizando a linguagem de programação C++. A topologia de rede utilizada é em grade, sendo composta por dez nós que estão a uma distância de $500 \mathrm{~m}$ entre si. Para a cobertura da distância, é utizado um ganho de potência nos nós, de modo que o raio de cobertura de cada nó seja de $656 \mathrm{~m}$.

$\mathrm{Na}$ simulação, são gerados, aleatoriamente, três canais, utilizando uma distribuição uniforme em cada nó. Com isso, cada canal tem a mesma probabilidade de ser gerado. Os canais que podem ser gerados são o 1, 6 e 11 do padrão IEEE 802.11b. Esses canais foram escolhidos para respeitar a distância mínima de $25 \mathrm{MHz}$ entre canais, de modo a evitar a interferência entre eles.

A interface de controle e de dados possuem os endereços de rede 10.1.1.0/24 e 10.1.2.0/24, respectivamente.

A técnica de modulação empregada é a DSSS com modelo de atraso de propagação ConstantSpeedPropagationDelayModel e o modelo de perda de propagação é o FriisPropagationLossModel. Esses modelos foram escolhidos, pois são os utilizados nos exemplos-padrões do simulador. No entanto, poderia ser utilizado qualquer outro, desde que garantam um raio de cobertura maior que $500 \mathrm{~m}$ e menor que $1000 \mathrm{~m}$ para que as mensagens de hello não alcancem um vizinho a dois saltos. A Figura 8 ilustra a topologia de rede utilizada para o cenário de simulação.

Figura 8: Topologia do cenário de simulação

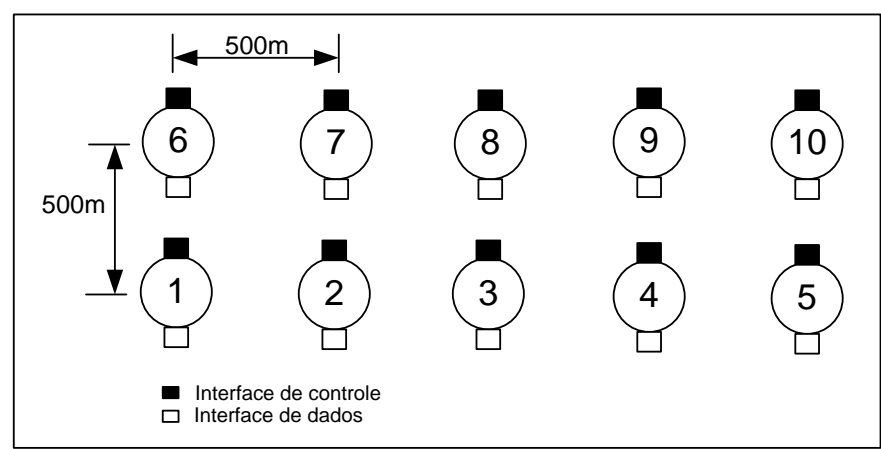


Como o raio de cobertura de cada nó é de $656 \mathrm{~m}$ e a distância entre os nós é de $500 \mathrm{~m}$, as mensagens de hello tem um alcance de 1 salto, não interferindo nos vizinhos a 2 saltos. O tempo de simulação é de 12 segundos.

\section{Resultados}

Para cada par de nós $\mathrm{X}-\mathrm{Y}$, onde $\mathrm{X}$ representa um nó e $\mathrm{Y}$ o seu vizinho e $\{\mathrm{a}, \mathrm{b}, \mathrm{c}, \ldots \mathrm{k}\}$ representa a lista de canais de um nó, com $\{\mathrm{a}, \mathrm{b}, \mathrm{c} \ldots \mathrm{k}\} \in \mathrm{N}^{*}$, tem-se que haverá uma conexão entre dois nós (enlace ativo) se $\mathrm{X}\left\{\mathrm{a}_{1}, \mathrm{~b}_{1}, \mathrm{c}_{1} \ldots \mathrm{k}_{1}\right\} \cap \mathrm{Y}\left\{\mathrm{a}_{2}, \mathrm{~b}_{2}, \mathrm{c}_{2} \ldots \mathrm{k}_{2}\right\} \neq \varnothing$. Após a simulação, obteve-se os seguintes conjuntos de canais disponíveis para os enlaces: $1-2=\{6,11\}, 2-3=\{11\}, 3-4=\{6\}, 4-5=\{6,11\}, 6-1=\{6,11\}, 6-7=\{6,11\}, 7-8=\{6,11\}, 8-9=\{\}, 9$ $10=\{1\}, 7-2=\{6,11\}, 8-3=\{1,11\}, 9-4=\{6\}, 10-5=\{11\}$.

A Tabela 1 ilustra o repositório criado com os pares de nós e os respectivos canais disponíveis. Cada par de nós representa duas estações na topologia de rede da Figura 8.

Tabela 1: Repositório de canais disponíveis para cada par de nós
\begin{tabular}{|c|c|}
\hline Par de nó (X-Y) & $\begin{array}{c}\text { Canais disponíveis } \\
\{\mathrm{a}, \mathrm{b}, \mathrm{c}, \ldots \mathrm{k}\}\end{array}$ \\
\hline $1-2$ & 6,11 \\
\hline $2-3$ & 11 \\
\hline $3-4$ & 6 \\
\hline $4-5$ & 6,11 \\
\hline $6-1$ & 6,11 \\
\hline $6-7$ & 6,11 \\
\hline $7-8$ & 6,11 \\
\hline $8-9$ & $*$ \\
\hline $9-10$ & 1 \\
\hline $7-2$ & 6,11 \\
\hline $8-3$ & 1,11 \\
\hline $9-4$ & 6 \\
\hline $10-5$ & 11 \\
\hline Não há canais disponíveis, pois os nós 8 e 9 não possuem \\
nenhum canal de frequência que sejam iguais entre si
\end{tabular}

Com a geração de três canais aleatórios, foram obtidas sete combinações de nós que podem selecionar um entre dois canais, possibilitando a comunicação simultânea entre pares de nós. Dessa forma, o par 1-2, representado pelos dispositivos 1 e 2 na Figura 8, pode utilizar o canal 6 enquanto o par 6-7 utiliza o 11, evitando a interferência entre enlaces devido à utilização do mesmo canal. Pode-se observar que o par 9-10 tem somente um único canal disponível (canal 1). Um dos pares vizinhos é o 4-5 que possui os canais 6 e 11 disponíveis. Como os canais entre os dois enlaces são diferentes, pode ser selecionado qualquer um dos canais que não causará interferência no par 9-10.

Observa-se também que foram obtidas cinco combinações de pares de nós com um único canal, sendo, portanto, a única opção de uso. Nesse caso, se os pares vizinhos do nó tiverem disponível o mesmo canal, não haverá como resolver o problema de interferência devido à falta de disponibilidade de canais diferentes (há somente um canal disponível em cada par de nós e eles são iguais). Caso o par de nós vizinhos tenha um canal diferente, não haverá problemas de interferências (par de nós vizinhos com um único canal, porém diferentes).

Além disso, observa-se que no par 8-9 não houve nenhum canal comum entre os dois nós, tornando o enlace inativo. Como cada nó desse par gerou um conjunto diferente de canais disponíveis, a intersecção de ambos os conjuntos, resultou em um conjunto vazio. Nesse caso, a conexão poderá ser reestabelecida após a próxima troca de mensagens de hello, desde que os dois nós possuam no mínimo um canal em comum. Uma 
opção para contornar o problema da desconexão devido à ausência de canais comuns entre dois nós, é disponibilizar pelo menos um canal que seja comum a todos os nós. O custo dessa opção é a maior probabilidade de colisão e interferência, porém o enlace sempre estará ativo.

\section{Conclusão}

Uma rede sem fio IEEE $802.11 \mathrm{~b} / \mathrm{g}$ convencional opera em um número de canal fixo no qual não há um repositório com os valores de canais disponíveis dos nós vizinhos. Com o protocolo apresentado obtém-se esse repositório, permitindo a tomada de decisão para a seleção de canais que sejam menos interferentes. Além de permitir a seleção de canais que sejam menos interferentes, a atribuição de canais diferentes para cada enlace, permite a transmissão simultânea de dados na rede, aumentando a sua vazão. O protocolo não se limita somente ao padrão IEEE $802.11 \mathrm{~b} / \mathrm{g}$, podendo ser mapeado para outras faixas de frequência. Em trabalhos futuros, o protocolo poderá ser extendido para a implementação de um algoritmo de seleção de canais que seja menos interferente, a implementação de mensagens para a solicitação de comutação de canais e a integração desse protocolo com protocolos de roteamento.

\section{Agradecimentos}

Os autores agradecem à Unipar pelo apoio financeiro.

\section{Referências}

[1] ZHANG, Y. et al. Wireless mesh networking: architectures, protocols and standards. New York: Auerbach Publications, 2007

[2] SUBRAMANIAN, A; GUPTA, H. Minimum-interference channel assignment in multi-radio wireless mesh networks. Disponível em: <http://www.cs.sunysb.edu/ hgupta/ps/channel.pdf>. Acesso em: 07 mar. 2012.

[3] GAST, M. Wireless networks: the definitive guide. Sebastopol: O’Reilly Media, Inc, 2005.

[4] FLICKENGER, R. Wireless networking in the developing world. NSRC. Disponível em: < http://wndw.net>. Acesso em: 05 mar. 2012.

[5] HOSSAIN, E.; NIYATO, D. Dynamic spectrum access and management in cognitive radio networks. Cambridge: Cambridge Universy Press, 2009.

[6] KHALIFE, H.; AHUJA, S. Joint routing and spectrum selection for multihop cognitive radio Networks. Disponível em: <http://citeseerx.ist.psu.edu/viewdoc/download?doi=10.1.1.218.8773\&rep=rep1\&type=pdf $>$. Acesso em: 07 mar. 2012.

[7] HURLEY, S.; SMITH, D. Meta-heuristics and channel assignment. Oxford: Oxford University Press, 2002. p. 22-44.

[8] SHIN, M.; SEUNGJOON L.; KIM, Y. Distributed channel assignment for multi-radio wireless networks. Proceedings In Mobile Adhoc and Sensor Systems (MASS). IEEE International Conference, 2006. Disponível em: <http://www2.research.att.com/ slee/pubs/safe-mass06.pdf $>$. Acesso em: 01 jul. 2012.

[9] ZHENG, C. et al. Maximum flow-segment basead channel assignment and routing in cognitive radio networks. Vehicular Technology Conference (VTC Sprint). Budapest, Hungary. IEEE Conference, 2011. Disponível em: <http://ieeexplore.ieee.org/xpls/abs_all.jsp?arnumber=5956172>. Acesso em: 05 jul. 2012

[10] WALENGA, P. et al. ZAP: Um algoritmo de atribuição distribuída de canais para mitigação de interferências em redes com rádio cognitivo. Anais do XXVIII Simpósio Brasileiro de Redes de Computadores e Sistemas Distribuídos. Gramado: SBRC, 2010. p. 175-188. 